\title{
All-Femtosecond Laser Keratorefractive Surgery
}

\author{
Anders Ivarsen $\cdot$ Jesper Hjortdal
}

Published online: 13 December 2013

(C) Springer Science + Business Media New York 2013

\begin{abstract}
During the last few years, refractive lenticule extraction (ReLEx) has been introduced as a new approach in the field of keratorefractive surgery. In contrast to previous treatments, ReLEx is an all-femtosecond laser treatment where a refractive lenticule is created within the intact cornea. The lenticule can be removed using a flapbased approach (ReLEx flex), but in its latest evolution (ReLEx smile), the lenticule is extracted through a small peripheral incision, leaving most of the anterior stroma untouched. In theory, this approach should spare most of the corneal nerves and minimize the biomechanical impact of the procedure. Clinical studies have shown the safety and the visual and refractive outcomes after ReLEx to be as good as after FS-LASIK. The current article aims to provide an up-to-date overview of all-femtosecond laser keratorefractive surgery with particular emphasis on the most current of approach, ReLEx smile.
\end{abstract}

Keywords Myopia - Astigmatism - Laser in situ keratomileusis (LASIK) · Keratorefractive surgery · Femtosecond laser - Refractive lenticule extraction (ReLEx)

\section{Introduction}

Excimer Laser Keratorefractive Surgery

During the last 20 years, the excimer laser has dominated the field of keratorefractive surgery, initially with surface

\footnotetext{
A. Ivarsen $(\bowtie) \cdot J$. Hjortdal

Department of Ophthalmology, Aarhus University Hospital, Noerrebrogade 44, 8000 Aarhus C, Denmark

e-mail: ai@dadlnet.dk
}

ablation procedures such as photorefractive keratectomy (PRK) and later with the flap-based laser in situ keratomileusis (LASIK). Due to excellent patient satisfaction, high precision, and very good safety [1, 2], LASIK has become one of the most frequently performed surgical procedures worldwide. LASIK is performed as a two-stage procedure, which involves the cutting of a flap in the anterior stroma followed by excimer laser photoablation of stromal tissue. Within the last decade, femtosecond lasers have mostly replaced manual microkeratomes for cutting of the LASIK flap (FS-LASIK). Although this may have improved the clinical outcome [3], the procedure still has potential weaknesses. First, flap-related complications such as traumatic flap dislocation [4], reduced corneal sensitivity due to severed stromal nerves [5], and surgically induced ectasia due to loss of biomechanical strength [6] remain significant challenges. Second, several factors may influence the precision of the photoablative procedure, including corneal hydration, room humidity, patient age, parallax error, and laser fluency [7, 8]. Furthermore, in surface ablation procedures, postoperative wound healing may cause stromal haze formation and affect the long-term stability of the obtained refractive correction, with myopic regression as a well-known complication of high myopic corrections [9].

\section{All-Femtosecond Laser Keratorefractive Surgery}

Within the last few years, surgical extraction of a refractive lenticule, or ReLEx ${ }^{\circledR}$, has evolved as a new treatment in the field of keratorefractive surgery. Presently, the Visumax ${ }^{\circledR}$ femtosecond laser (Carl Zeiss Meditec) is the only platform to offer this treatment. The 500-kHz Visumax laser generates very fast pulses $\left(10^{-15} \mathrm{~s}\right)$ in the near-infrared spectrum. Depending on the specific laser settings, each pulse 
conveys approximately $150 \mathrm{~nJ}$, which causes localized photodisruption at the focal point. The generated plasma expands, creating a cavitation bubble and, as individual cavitation bubbles fuse, the stroma is cut with a minimum of collateral damage. The Visumax uses a high numerical aperture, concave contact glass to focus the laser pulses with very high precision. Thus, laser spots of approximately $1-\mu \mathrm{m}$ diameter are placed with a defined distance of $2-5 \mu \mathrm{m}$ in a spiral pattern. To ensure centration on the visual axis, the patient fixates on a blinking light, and suction is applied at the limbus to maintain stability of the eye. Initially, the posterior surface of the lenticule is cut, followed by creation of the anterior surface, which is slightly enlarged in diameter to facilitate surgical manipulation. Depending on the method used to access the lenticule, ReLEx can be divided into ReLEx flex, in which a LASIK-like flap allows surgical removal of the lenticule, and ReLEx smile, in which a small incision (approximately 2-4 mm in length) is created for manual lenticule extraction. A blunt spatula is used to break any remaining tissue bridges after the laser treatment, and the lenticule is grasped and removed with a pair of forceps.

For further details on the surgical approach, please refer to Sekundo et al. [10], Shah et al. [11], and Vestergaard et al. [12].

In contrast to LASIK, ReLEx represents a one-laser approach, where the critical laser treatment is performed on the intact cornea rather than on exposed corneal stroma. Consequently, the potential variability associated with the excimer laser photoablation is avoided. In addition, the minimally invasive ReLEx smile treatment have several theoretical advantages over flap-based treatments, including very little trauma to the corneal surface, less corneal denervation, and better biomechanical strength due to an almost intact anterior stroma. Since the first introduction of ReLEx, the repetition rate of the Visumax laser has been increased from 200 to $500 \mathrm{kHz}$, and the settings for laser spot size, energy, and distance have been optimized, changes that may have had a significant impact on the clinical outcome after surgery. Furthermore, the flap-based ReLEx flex represents an evolutionary step before ReLEx smile and is today primarily used as an introductory step for new ReLEx surgeons. Due to these changes, the present review focuses primarily on studies concerning ReLEx smile. However, the number of clinical publications on ReLEx is still very limited, with only approximately five papers reporting on the outcome after ReLEx smile and fewer than 20 papers investigating ReLEx flex, many of which consider the initial $200-\mathrm{kHz}$ Visumax. Thus, where appropriate, significant studies on ReLEx flex are incorporated.

At present, the Visumax allows myopic corrections up to -10 diopters (D) spherical equivalent (SE) correction, with an astigmatic component of up to 5 D. Hyperopic treatments are not available at the moment, although one study has reported on the outcome of hyperopic ReLEx flex [13]. The Visumax laser is CE (Conformité Européenne) marked and is currently being evaluated in clinical studies for the approval of ReLEx by the FDA (US Food and Drug Administration).

\section{Clinical Results}

\section{Refractive Outcome}

Overall, ReLEx has been reported to have high refractive predictability (Table 1). In the largest report to date on ReLEx smile in 670 myopic eyes 3 months after surgery, the mean error in SE refraction was $-0.25 \pm 0.44 \mathrm{D}$, with $80 \%$ of eyes within $\pm 0.50 \mathrm{D}$ and $94 \%$ within $\pm 1.0 \mathrm{D}$ [14••]. We recently extended this evaluation to the first 1,574 eyes 3 months after ReLEx smile and found a similar mean error of $-0.15 \pm 0.50 \mathrm{D}$ with $77 \%$ of eyes within $\pm 0.50 \mathrm{D}$ and $95 \%$ within $\pm 1.0 \mathrm{D}$ [31]. Other reports on ReLEx smile $[11,12,15,16]$ and $500-\mathrm{kHz}$ ReLEx flex [17-21] have found very similar refractive outcomes in smaller numbers of patients.

The refractive stability after ReLEx smile has not been extensively investigated. However, in one study on 279 eyes with high myopia, refraction was found to be stable from 1 to 3 months after surgery, although a minor regression of $-0.15 \mathrm{D}$ was observed during the first month [12]. One other study on 54 eyes found no regression during the first 6 months after surgery [15]. Similarly, no regression has been found during the first 3-6 months after $500-\mathrm{kHz}$ ReLEx flex [17, 18, 20, 21] or for 1 year after 200-kHz ReLEx flex [22, 23].

Interestingly, the refractive predictability after ReLEx smile has been found to be unrelated to the degree of the attempted myopic correction [14••]. This stands in contrast to excimer-based treatments, which show decreasing precision with increasing myopic correction [24]. Furthermore, other parameters including preoperative corneal power, patient age, and gender have been found to have very limited impact on the refractive outcome after ReLEx smile [14••].

Correction of high astigmatism has not yet been systematically evaluated after ReLEx. Only one paper on 200-kHz ReLEx flex contains a rigorous evaluation of the outcome after cylinder correction [25]. An undercorrection of approximately $10 \%$ was reported; however, the average preoperative cylinder was only $0.96 \pm 0.87 \mathrm{D}$, and the population skewed toward low corrections, making it difficult to extrapolate to high astigmatisms. Recently, we evaluated correction of myopic astigmatism with ReLEx 


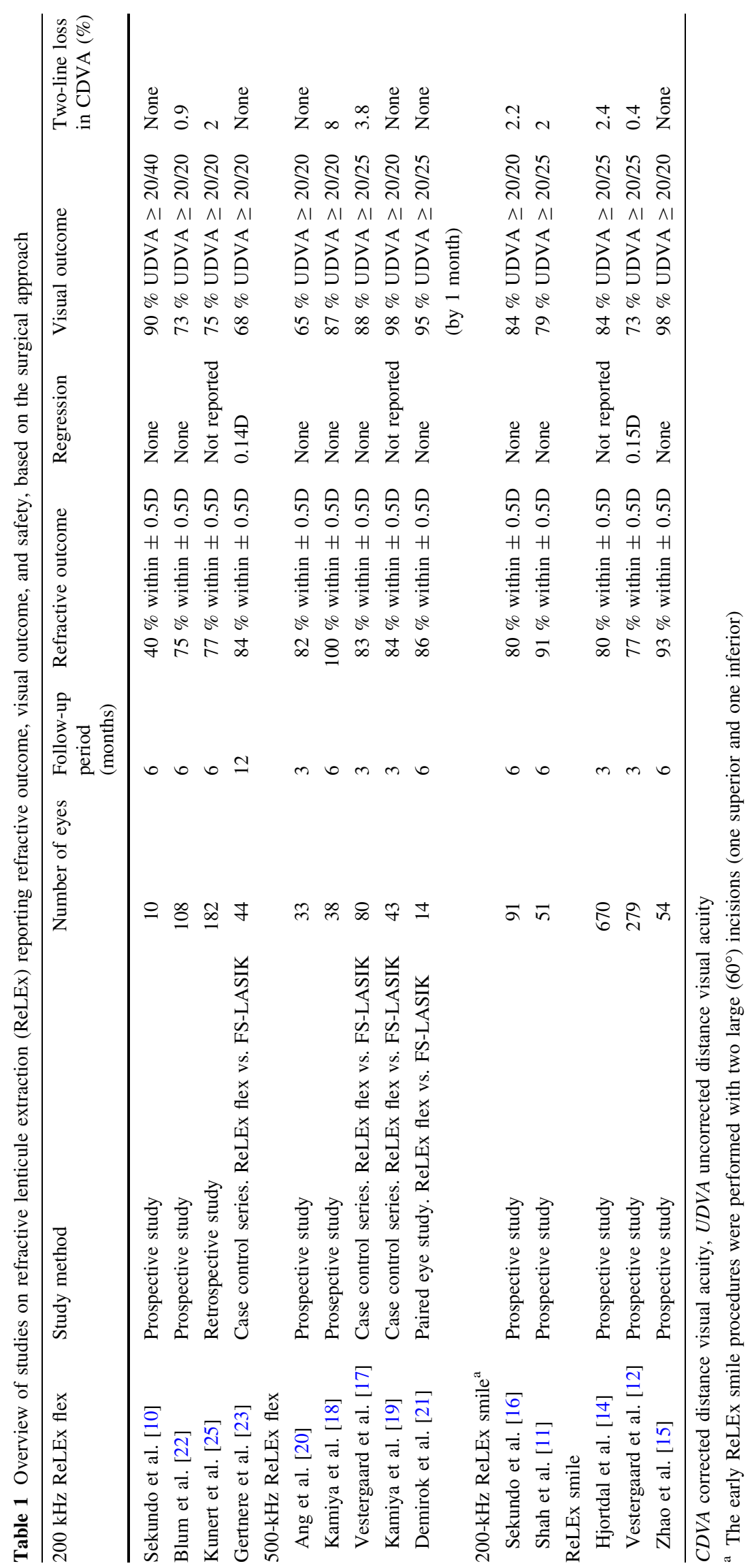


smile in 775 eyes, of which 106 eyes had an astigmatism of $2.50 \mathrm{D}$ or more. On average, $95 \%$ were within $\pm 1.0 \mathrm{D}$ of the attempted spherical equivalent correction three months after surgery. However, a significant astigmatic undercorrection was observed, with an average error of treatment of $0.17 \pm 0.42 \mathrm{D}$ in low astigmatism and $0.59 \pm 0.65 \mathrm{D}$ in high astigmatism [26].

At present, only one study has examined hyperopic treatment (average SE refraction $+2.8 \pm 1.3 \mathrm{D}$ ) with 200-kHz ReLEx flex [13]. After 9 months, only $64 \%$ of patients had a postoperative refraction within $\pm 1.0 \mathrm{D}$ of that attempted, and there was significant regression of the effect during the first 6 months after surgery. Thus, it still remains to be determined whether ReLEx eventually will allow safe and predictable hyperopic treatments.

\section{Visual Outcome}

In the first clinical studies, ReLEx flex was reported to have delayed visual recovery in comparison with FSLASIK [11, 27]. However, later studies suggested that the laser scanning pattern and energy had an impact on the lenticule surface quality and the immediate postoperative outcome $[28 \bullet, 29,30]$. Subsequent changes in the laser scanning trajectory and energy delivery appear to have eliminated the problem with postoperative visual recovery.

Three studies have examined the uncorrected distance visual acuity (UDVA) after 500-kHz ReLEx smile for myopia and report 73-100 \% of patients as having an UDVA of 20/25 or better 3-6 months after surgery (Table 1) [12, 14••, 15]. Similar results have been reported for $500-\mathrm{kHz}$ ReLEx flex [17-21], and the procedure has been found to be on par with the outcome after FS-LASIK [17, 21]. The efficacy index 3 months after ReLEx smile (postoperative UDVA/preoperative CDVA) has been found to be $0.90 \pm 0.25$, indicating that a patient on average can expect a postoperative UDVA of $90 \%$ of their preoperative CDVA [14••].

\section{Safety and Complications}

The induced change in corrected distance visual acuity (CDVA) may be used as an indicator for the overall safety of a refractive surgical procedure. In general, loss or gain of two or more lines on the Snellen visual acuity card is considered significant and noticeable for the patient.

Most existing studies on ReLEx flex or ReLEx smile are too small to properly evaluate the safety of the procedure, and the frequency of a two-line loss in CDVA has been reported to lie between 0 and $8 \%$ (Table 1) [10,11, 15-21]. In one study on 279 eyes after ReLEx smile, $0.4 \%$ of eyes were reported to have a loss of two or more lines [12]. In contrast, a $2.4 \%$ risk of a two-line loss was found in 670 eyes by Hjortdal et al. [14••]. However, in the same study, a safety index (CDVA before/CDVA after surgery) of $1.07 \pm 0.22$ was found, indicating that CDVA on average increased after surgery, as would be expected because of the image magnification of myopic keratorefractive procedures. In a recent single-center study, the safety and complications of 1,574 ReLEx smile procedures were evaluated after 3 months [31]. CDVA was found to have improved with two or more lines in $3.4 \%$ of eyes, whereas $1.5 \%$ of eyes had experienced a loss of two or more lines. Yet, at a late follow-up visit, all patients with a loss in visual acuity had recovered to within one line of the preoperative value. The surgeon learning curve and the laser settings were found to be important parameters for the postoperative visual recovery. Overall, safety after ReLEx smile appears to be on par with that reported after FS-LASIK [1, 3], although recovery may be prolonged in a few cases.

Endothelial changes after ReLEx have not been systematically evaluated. Only one study on 38 eyes has reported endothelial cell counts and found that ReLEx flex induced no significant changes in endothelial cell density [18].

A variety of peri- and postoperative complications have been reported after ReLEx smile. The most frequently reported perioperative complications include tears at the incision and minor abrasions, whereas decentration, suction loss, difficulties removing the lenticule, and cap perforation may rarely occur $[11,12,16,17,25]$. Frequent postoperative complications include dry eye, microstriae, and increased interface scatter, whereas rare complications include keratitis, interface inflammation, epithelial ingrowth, and monocular ghost images [17, 18, 25]. Recently, we found irregular postoperative topography in 18 of 1,574 eyes, giving rise to ghost images in six cases [31]. Topography-guided PRK was performed in four of these eyes, ameliorating the symptoms in three cases. In another recent paper, a lenticule remnant was documented to be the cause of postoperative monocular double vision [32]. Postoperative ectasia has not been reported after ReLEx smile; however, one case has been documented after a flap-based ReLEx flex treatment [22].

Overall, ReLEx smile appears to be a technically more demanding surgical procedure than LASIK, introducing new potential complications related to the extraction of the refractive lenticule. Still, despite a relatively high frequency of peri- and postoperative complications, the visual outcome is reported to be good, with minimal risk of loss in CDVA on the long term.

Higher Order Aberrations (HOAs) and Contrast Sensitivity

Only three studies have compared the induced HOAs after ReLEx flex and LASIK [19] or FS-LASIK [17, 23]. In these studies, both ReLEx flex and LASIK were found to 
increase the total corneal or whole-eye HOAs; however, ReLEx flex induced less spherical aberration than LASIK. Furthermore, in LASIK the induced HOAs were found to increase with the degree of attempted refractive correction, whereas in ReLEx flex no such correlation was found [19]. Parallax error during excimer laser photoablation has been suggested as an explanation for the observed differences in induced spherical aberration after LASIK and ReLEx flex [19]. As of yet, no studies have reported changes in HOAs after ReLEx smile with the $500-\mathrm{kHz}$ Visumax laser.

Contrast sensitivity after ReLEx has been examined in one retrospective, comparative study on 200-kHz ReLEx flex and FS-LASIK. Both procedures showed a similar increase in photopic contrast sensitivity after 1 year; however, ReLEx flex also showed an improvement in mesopic contrast that was not found after FS-LASIK [23]. Presently, changes in contrast sensitivity have not been evaluated after ReLEx smile.

Based on the relatively few comparative studies on HOAs and contrast sensitivity, ReLEx appears to be on par with or better than LASIK; however, studies on ReLEx smile are lacking.

\section{Corneal Sensitivity and Tear Secretion}

Three studies have examined the corneal sensitivity after ReLEx smile in comparison with a flap-based treatment $[33 \cdot \bullet, 34,35]$. In a randomized paired-eye study, Demirok et al. demonstrated less reduction in corneal sensitivity after ReLEx smile than after FS-LASIK, although sensitivity had fully normalized in both groups by 6 months [34]. In another randomized paired-eye study, the corneal nerve density and number of long nerve fibers were higher after ReLEx smile than after ReLEx flex [33••]; accordingly, sensitivity was better after ReLEx smile. Finally, in a comparative study on ReLEx smile, ReLEx flex, and FSLASIK, better sensitivity was found at all time points for up to 3 months after ReLEx smile [35].

Two studies have performed a paired-eye evaluation of the postoperative tear secretion after ReLEx smile in comparison with a flap-based treatment [33••, 34]. In both studies, no significant differences in tear osmolarity, tear secretion rate, and tear meniscus height were observed. However, one study found a slight difference in the postoperative tear-film break-up time in favor of ReLEx smile [33••].

Overall, the minimally invasive ReLEx smile causes less damage to corneal nerves than flap-based treatments, resulting in better postoperative sensitivity. However, the nervous changes appear to have only minimal measurable impact on the postoperative tear secretion.
Corneal Biomechanics and Sublayer Thickness

In ReLEx smile, most of the anterior stroma remains intact after surgery. Since the cornea is biomechanically strongest in the anterior part [36], it would theoretically be more robust after ReLEx smile than after a flap-based treatment where most of the anterior lamellae are severed. Thus, ReLEx smile-treated corneas may be hypothesized to be more resistant to trauma and less prone to developing postoperative keratectasia, and they have been suggested to be even stronger than PRK-treated corneas [37•]. Furthermore, it has recently been speculated that the refractive lenticule should be removed deeper within the stroma to increase the postoperative corneal strength [37•]. Although this might be advantageous from a biomechanical point of view, many factors could affect the outcome, including endothelial safety, the quality of the laser cut in deeper stroma, and the relative front- and back-surface changes. Thus, the optimal depth of the refractive lenticule still remains to be determined.

At present, only one study has been published on the biomechanical properties after ReLEx smile in comparison with FS-LASIK using the Ocular Response Analyzer [38]. This paired-eye, randomized study found no differences in corneal hysteresis $(\mathrm{CH})$ or corneal resistance factor (CRF) 6 months after surgery. In a comparable study on ReLEx smile and ReLEx flex, we similarly found no difference between the methods regarding $\mathrm{CH}$ and CRF [39]. However, in a small comparative study on ReLEx smile, ReLEx flex, and FS-LASIK, we recently found the biomechanical response, as measured with the Corvis ST, to be more abnormal after a flap-based treatment than after ReLEx smile [40]. Overall, the biomechanical changes after ReLEx smile are still unclear, and there is a considerable need for further studies.

A planar and uniform flap is generally considered important in flap-based keratorefrative surgery, and three studies have found the cap to be of nearly uniform thickness and similar to the flap after ReLEx flex or FS-LASIK [15, 41, 42]. Furthermore, in one study, no significant changes were observed in central cap or stromal bed thickness for 6 months after surgery [42]. We recently evaluated corneal sublayer thicknesses after ReLEx smile and ReLEx flex [39] and found no significant difference in cap or stromal bed thickness 6 months after surgery. However, as seen after other myopic keratorefractive procedures [43, 44], a compensatory epithelial hyperplasia was observed.

\section{Retreatment}

Although ReLEx has been found to have a high refractive predictability, some patients have a postoperative residual 
refractive error due to over- or undercorrection [14••]. In flap-based treatments, an excimer-based enhancement procedure can be performed after lifting the flap [45]. However, retreatment after ReLEx smile is more complicated.

Possible approaches may include PRK or LASIK, whereas a new ReLEx procedure may be more unpredictable due to multiple dissection planes within the cornea. Presently, no systematic clinical evaluation of ReLEx smile enhancements has been published. However, one study has reported on successful topography-guided PRK in ReLEx smile patients with postoperative irregular astigmatism [31], and another has reported on successful FS-LASIK in a patient with perioperative suction loss [46]. Furthermore, in a rabbit model, the conversion of a ReLEx smile cap to a flap has been demonstrated, allowing subsequent intrastromal photoablation [47]. Still, the optimal approach for ReLEx smile enhancements needs to be established.

\section{Experimental Studies}

In excimer laser keratorefractive surgery, the energy delivered to the cornea may promote subsequent inflammation and wound repair [48]. In contrast to the excimer laser, femtosecond lasers deliver only minimal amounts of energy to surrounding tissue [49], suggesting that the femtosecond laser may induce less postoperative wound repair. In accordance, a recent study in rabbits has demonstrated ReLEx flex to induce less wound healing and inflammation than FS-LASIK, particularly after high myopic corrections [49]. Whether this observation has any clinical consequence remains to be determined; however, wound repair after LASIK and, in particular, PRK has been extensively investigated and is known to influence the postoperative outcome [48, 50, 51].

Extracting an intact stromal lenticule from the cornea opens new interesting possibilities in keratorefractive surgery. First, if the extracted tissue can be successfully preserved, the surgical procedure may in theory be reversed at a later time point by reimplantation of the lenticule. Second, the lenticule could, speculatively, be used to change the refraction in another individual, for example, by implanting a myopic lenticule in the stromal pocket of a hyperopic patient, a procedure that in Europe at least would require permission from national authorities. In a recent study, refractive lenticules from rabbit eyes were demonstrated to have an intact collagen structure and viable keratocytes after 1 month cryopreservation [52]. Other studies in rabbits and primates have shown successful cryopreservation and later reimplantation of a stromal lenticule [53-55]. Furthermore, in primates, the procedure was shown to induce little postoperative wound repair, and keratocyte repopulation could be observed after 16 weeks [46]. Thus, although further studies are needed, lenticule reimplantation or transplantation from one patient to another may become reality in the near future.

\section{Conclusion}

Several studies have shown that the refractive and visual outcomes after ReLEx smile and ReLEx flex are as good as after FS-LASIK, and ReLEx has even been indicated to induce fewer HOAs. Furthermore, ReLEx smile has been shown to be as safe as LASIK, although the procedure may be technically more demanding and have a different variety of complications.

The minimal impact on the anterior stroma in ReLEx smile represents the most interesting aspect of the new procedure. Thus, stromal nerves are spared, and ReLEx smile has been convincingly demonstrated to cause less denervation and have better sensitivity than flap-based treatments. Yet, the impact on postoperative tear secretion and dry-eye symptoms remains unclear. Due to the intact anterior stromal lamellae, the cornea may be stronger after ReLEx smile than after a flap-based treatment. However, biomechanical differences have proven elusive and have not yet been positively confirmed.

In its current state, ReLEx smile has been shown to be a reliable, efficient, and safe procedure for myopic corrections. Correction of myopic astigmatism also appears promising, but hyperopic treatments need further evaluation, and the long-term outcome and biomechanical properties of ReLEx smile remain undetermined. Furthermore, compensation for eye rotation as well as aspheric or custom lenticule profiles is still not available. Thus, in complicated cases with irregular corneas, the excimer laser is still the only valid option. In contrast, ReLEx may allow exciting new treatments including reimplantation or transplantation of refractive lenticules, and it is of considerable interest to see the further evolution of ReLEx over the coming years.

Disclosure Anders Ivarsen declares that he has no conflict of interest. Jesper Hjortdal received travel reimbursement from Carl Zeiss Meditec.

Human and Animal Rights and Informed Consent The article does not contain any studies with human or animal subjects performed by any of the authors. 


\section{References}

Papers of particular interest, published recently, have been highlighted as:

- Of importance,

-• Of major importance

1. Tomita M, Waring GO 4th, Magnago T, Watabe M. Clinical results of using a high-repetition-rate excimer laser with an optimized ablation profile for myopic correction in 10235 eyes. J Cataract Refract Surg. 2013;39(10):1543-9.

2. Brown MC, Schallhorn SC, Hettinger KA, Malady SE. Satisfaction of 13,655 patients with laser vision correction at 1 month after surgery. J Refract Surg. 2009;25(7 Suppl):S642-6.

3. Farjo AA, Sugar A, Schallhorn SC, Majmudar PA, Tanzer DJ, Trattler WB, Cason JB, Donaldson KE, Kymionis GD. Femtosecond lasers for LASIK flap creation: a report by the American Academy of Ophthalmology. Ophthalmology. 2013;120(3): e5-20.

4. Iskander NG, Peters NT, Anderson Penno E, Gimbel HV. Late traumatic flap dislocation after laser in situ keratomileusis. J Cataract Refract Surg. 2001;27(7):1111-4.

5. Pérez-Santonja JJ, Sakla HF, Cardona C, Chipont E, Alió JL. Corneal sensitivity after photorefractive keratectomy and laser in situ keratomileusis for low myopia. Am J Ophthalmol. 1999;127(10):497-504.

6. Geggel HS, Talley AR. Delayed onset keratectasia following laser in situ keratomileusis. J Cataract Refract Surg. 1999;25(4): 582-6.

7. Ang EK, Couper T, Dirani M, Vajpayee RB, Baird PN. Outcomes of laser refractive surgery for myopia. J Cataract Refract Surg. 2009;35(5):921-33.

8. Walter KA, Stevenson AW. Effect of environmental factors on myopic LASIK enhancement rates. J Cataract Refract Surg. 2004;30(4):798-803.

9. Vestergaard AH, Hjortdal JØ, Ivarsen A, Work K, Grauslund J, Sjølie AK. Long-term outcomes of photorefractive keratectomy for low to high myopia: 13 to 19 years of follow-up. J Refract Surg. 2013;29(5):312-9.

10. Sekundo W, Kunert K, Russmann C, Gille A, Bissmann W, Stobrawa G, Sticker M, Bischoff M, Blum M. First efficacy and safety study of femtosecond lenticule extraction for the correction of myopia: six-month results. J Cataract Refract Surg. 2008;34(9):1513-20.

11. Shah R, Shah S, Sengupta S. Results of small incision lenticule extraction: all-in-one femtosecond laser refractive surgery. J Cataract Refract Surg. 2011;37(1):127-37.

12. Vestergaard A, Ivarsen AR, Asp S, Hjortdal JØ. Small-incision lenticule extraction for moderate to high myopia: predictability, safety, and patient satisfaction. J Cataract Refract Surg. 2012;38(11):2003-10.

13. Blum M, Kunert KS, Voßmerbäumer U, Sekundo W. Femtosecond lenticule extraction (ReLEx) for correction of hyperopia-first results. Graefes Arch Clin Exp Ophthalmol. 2013; 251(1):349-55.

14. $\bullet$ Hjortdal JØ, Vestergaard AH, Ivarsen A, Ragunathan S, Asp S. Predictors for the outcome of small-incision lenticule extraction for myopia. J Refract Surg. 2012;28(12):865-71. Large clinical study on preoperative parameters affecting the outcome of ReLEx smile.

15. Zhao J, Yao P, Li M, Chen Z, Shen Y, Zhao Z, Zhou Z, Zhou X. The morphology of corneal cap and its relation to refractive outcomes in femtosecond laser small incision lenticule extraction
(SMILE) with anterior segment optical coherence tomography observation. PLoS ONE. 2013;8(8):e70208.

16. Sekundo W, Kunert KS, Blum M. Small incision corneal refractive surgery using the small incision lenticule extraction (SMILE) procedure for the correction of myopia and myopic astigmatism: results of a 6 month prospective study. Br J Ophthalmol. 2011;95(3):335-9.

17. Vestergaard A, Ivarsen A, Asp S, Hjortdal JØ. Femtosecond (FS) laser vision correction procedure for moderate to high myopia: a prospective study of ReLEx $\left({ }^{\circledR}\right)$ flex and comparison with a retrospective study of FS-laser in situ keratomileusis. Acta Ophthalmol. 2013;91(4):355-62.

18. Kamiya K, Igarashi A, Ishii R, Sato N, Nishimoto H, Shimizu K. Early clinical outcomes, including efficacy and endothelial cell loss, of refractive lenticule extraction using a $500 \mathrm{kHz}$ femtosecond laser to correct myopia. J Cataract Refract Surg. 2012;38(11):1996-2002.

19. Kamiya K, Shimizu K, Igarashi A, Kobashi H, Komatsu M. Comparison of visual acuity, higher-order aberrations and corneal asphericity after refractive lenticule extraction and wavefrontguided laser-assisted in situ keratomileusis for myopia. $\mathrm{Br} \mathrm{J}$ Ophthalmol. 2013;97(8):968-75.

20. Ang M, Chaurasia SS, Angunawela RI, Poh R, Riau A, Tan D, Mehta JS. Femtosecond lenticule extraction (FLEx): clinical results, interface evaluation, and intraocular pressure variation. Invest Ophthalmol Vis Sci. 2012;53(3):1414-21.

21. Demirok A, Agca A, Ozgurhan EB, Bozkurt E, Celik U, Demircan A, Guleryuz NB, Cankaya Kİ, Yilmaz OF. Femtosecond lenticule extraction for correction of myopia: a 6 month follow-up study. Clin Ophthalmol. 2013;7:1041-7.

22. Blum M, Kunert KS, Engelbrecht C, Dawczynski J, Sekundo W. Femtosecond lenticule extraction (FLEx)—results after 12 months in myopic astigmatism. Klin Monbl Augenheilkd. 2010;227(12):961-5.

23. Gertnere J, Solomatin I, Sekundo W. Refractive lenticule extraction (ReLEx flex) and wavefront-optimized Femto-LASIK: comparison of contrast sensitivity and high-order aberrations at 1 year. Graefes Arch Clin Exp Ophthalmol. 2013;251(5):1437-42.

24. Gazieva L, Beer MH, Nielsen K, Hjortdal J. A retrospective comparison of efficacy and safety of 680 consecutive lasik treatments for high myopia performed with two generations of flying-spot excimer lasers. Acta Ophthalmol. 2011;89(8):729-33.

25. Kunert KS, Russmann C, Blum M, Sluyterman VLG. Vector analysis of myopic astigmatism corrected by femtosecond refractive lenticule extraction. J Cataract Refract Surg. 2013;39(5):759-69.

26. Ivarsen A, Hjortdal J. Correction of myopic astigmatism with small incision lenticule extraction. J Refract Surg. (submitted).

27. Blum M, Kunert K, Schröder M, Sekundo W. Femtosecond lenticule extraction for the correction of myopia: preliminary 6-month results. Graefes Arch Clin Exp Ophthalmol. 2010; 248(7):1019-27.

28. - Shah R, Shah S. Effect of scanning patterns on the results of femtosecond laser lenticule extraction refractive surgery. J Cataract Refract Surg. 2011;37(9):1636-47. Clinical study emphasizing the importance of laser settings for the postoperative clinical outcome.

29. Riau AK, Ang HP, Lwin NC, Chaurasia SS, Tan DT, Mehta JS. Comparison of four different VisuMax circle patterns for flap creation after small incision lenticule extraction. J Refract Surg. 2013;29(4):236-44.

30. Kunert KS, Blum M, Duncker GI, Sietmann R, Heichel J. Surface quality of human corneal lenticules after femtosecond laser surgery for myopia comparing different laser parameters. Graefes Arch Clin Exp Ophthalmol. 2011;249(9):1417-24. 
31. Ivarsen A, Asp S, Hjortdal J. Safety and complications of more than 1500 small-incision lenticule extraction procedures. Ophthalmology. (accepted).

32. Dong Z, Zhou X. Irregular astigmatism after femtosecond laser refractive lenticule extraction. J Cataract Refract Surg. 2013;39(6):952-4.

33. • Vestergaard AH, Grønbech KT, Grauslund J, Ivarsen AR, Hjortdal JO. Subbasal nerve morphology, corneal sensation, and tear film evaluation after refractive femtosecond laser lenticule extraction. Graefes Arch Clin Exp Ophthalmol. 2013;251 (11):2591-600. Randomized paired-eye study directly demonstrating that ReLEx smile induces fewer changes in corneal nerve morphology and has better postoperative sensitivity than the flapbased ReLEx flex.

34. Demirok A, Ozgurhan EB, Agca A, Kara N, Bozkurt E, Cankaya KI, Yilmaz OF. Corneal sensation after corneal refractive surgery with small incision lenticule extraction. Optom Vis Sci. 2013;90(10):1040-7.

35. Wei S, Wang Y. Comparison of corneal sensitivity between FSLASIK and femtosecond lenticule extraction (ReLEx flex) or small-incision lenticule extraction (ReLEx smile) for myopic eyes. Graefes Arch Clin Exp Ophthalmol. 2013;251(6):1645-54.

36. Randleman JB, Dawson DG, Grossniklaus HE, McCarey BE, Edelhauser HF. Depth-dependent cohesive tensile strength in human donor corneas: implications for refractive surgery. J Refract Surg. 2008;24(1):S85-9.

37. - Reinstein DZ, Archer TJ, Randleman JB. Mathematical model to compare the relative tensile strength of the cornea after PRK, LASIK, and small incision lenticule extraction. J Refract Surg. 2013;29(7):454-60. Theoretical considerations on the biomechanical importance of various keratorefractive procedures.

38. Agca A, Ozgurhan EB, Demirok A, Bozkurt E, Celik U, Ozkaya A, Cankaya I, Yilmaz OF. Comparison of corneal hysteresis and corneal resistance factor after small incision lenticule extraction and femtosecond laser-assisted LASIK: a prospective fellow eye study. Cont Lens Anterior Eye. 2013;. doi:10.1016/j.clae.2013. 05.003 .

39. Vestergaard A, Grauslund J, Ivarsen A, Hjortdal J. Central corneal sublayer pachymetry and biomechanical properties after refractive femtosecond laser lenticule extraction. J Refract Surg. (accepted).

40. Bach-Pedersen I, Bak-Nielsen S, Vestergaard A, Ivarsen A, Hjortdal J. Corneal biomechanical properties after LASIK, ReLEx flex, and ReLEx smile by Scheimpflug-based dynamic tonometry. Graefes Arch. (submitted).

41. Ozgurhan EB, Agca A, Bozkurt E, Gencer B, Celik U, Cankaya KI, Demirok A, Yilmaz OF. Accuracy and precision of cap thickness in small incision lenticule extraction. Clin Ophthalmol. 2013;7:923-6.

42. Tay E, Li X, Chan C, Tan DT, Mehta JS. Refractive lenticule extraction flap and stromal bed morphology assessment with anterior segment optical coherence tomography. J Cataract Refract Surg. 2012;38(9):1544-51.

43. Ivarsen A, Fledelius W, Hjortdal JØ. Three-year changes in epithelial and stromal thickness after PRK or LASIK for high myopia. Invest Ophthalmol Vis Sci. 2009;50(5):2061-6.

44. Patel SV, Erie JC, McLaren JW, Bourne WM. Confocal microscopy changes in epithelial and stromal thickness up to 7 years after LASIK and photorefractive keratectomy for myopia. J Refract Surg. 2007;23(4):385-92.

45. Netto MV, Wilson SE. Flap lift for LASIK retreatment in eyes with myopia. Ophthalmology. 2004;111(7):1362-7.

46. Sharma R, Vaddavalli PK. Implications and management of suction loss during refractive lenticule extraction (ReLEx). J Refract Surg. 2013;29(7):502-3.

47. Riau AK, Ang HP, Lwin NC, Chaurasia SS, Tan DT, Mehta JS. Comparison of four different VisuMax circle patterns for flap creation after small incision lenticule extraction. J Refract Surg. 2013;29(4):236-44.

48. Alio JL, Javaloy J. Corneal inflammation following corneal photoablative refractive surgery with excimer laser. Surv Ophthalmol. 2013;58(1):11-25.

49. Riau AK, Angunawela RI, Chaurasia SS, Lee WS, Tan DT, Mehta JS. Early corneal wound healing and inflammatory responses after refractive lenticule extraction (ReLEx). Invest Ophthalmol Vis Sci. 2011;52(9):6213-21.

50. Moller-Pedersen T, Cavanagh HD, Petroll WM, Jester JV. Stromal wound healing explains refractive instability and haze development after photorefractive keratectomy: a 1-year confocal microscopic study. Ophthalmology. 2000;107(7):1235-45.

51. Ivarsen A, Laurberg T, Møller-Pedersen T. Characterisation of corneal fibrotic wound repair at the LASIK flap margin. Br J Ophthalmol. 2003;87(10):1272-8.

52. Mohamed-Noriega K, Toh KP, Poh R, Balehosur D, Riau A, Htoon HM, Peh GS, Chaurasia SS, Tan DT, Mehta JS. Cornea lenticule viability and structural integrity after refractive lenticule extraction (ReLEx) and cryopreservation. Mol Vis. 2011;17:3437-49.

53. Riau AK, Angunawela RI, Chaurasia SS, Lee WS, Tan DT, Mehta JS. Reversible femtosecond laser-assisted myopia correction: a non-human primate study of lenticule re-implantation after refractive lenticule extraction. PLoS ONE. 2013;8(6):e67058.

54. Angunawela RI, Riau AK, Chaurasia SS, Tan DT, Mehta JS. Refractive lenticule re-implantation after myopic ReLEx: a feasibility study of stromal restoration after refractive surgery in a rabbit model. Invest Ophthalmol Vis Sci. 2012;53(8):4975-85.

55. Liu H, Zhu W, Jiang AC, Sprecher AJ, Zhou X. Femtosecond laser lenticule transplantation in rabbit cornea: experimental study. J Refract Surg. 2012;28(12):907-11. 